Pacific Journal of Mathematic 


\section{AZUMAYA ALGEBRAS OVER HENSEL RINGS}

\section{Rosario Strano}

In this paper we prove the following theorem.

Let $(R, \mathfrak{a})$ be an henselian couple and let $\mathscr{P}(R)$ be the set of isomorphism classes of Azumaya $R$-algebras; then the canonical map

$$
\mathscr{P}(R) \longrightarrow \mathscr{P}(R / \mathfrak{a})
$$

is bijective.

As a corollary we obtain that, if $(R, a)$ is an henselian couple, then the canonical homomorphism

$$
\mathscr{B}_{z}(R) \longrightarrow \mathscr{B}_{z}(R / \mathfrak{a})
$$

between the Brauer groups, is an isomorphism.

Introduction. The corollary mentioned in the abstract generalizes a theorem of Azumaya ([2], Th. 31). The proof is similar to the one used by Grothendieck in proving the above theorem in case that $R$ is a local ring and $a$ is its maximal ideal ([6], Th. 6.1).

Concerning the definition of henselian couple and Azumaya algebra we refer to [10] and [9] respectively.

All the rings and algebras are supposed to have unity.

In $\S 1$ we recall some properties of representable functors and smooth morphisms we shall need later.

In $\S \S 2,3$ we study two particular functors $F_{1}, F_{2}$ from the category of commutative $R$-algebras to the category of sets and we prove that $F_{1}$ and $F_{2}$ are represented by smooth commutative $R$-algebras. These functors will be used to prove the theorem.

In $\S 4$, applying a known property of henselian couples, we obtain the theorem stated before and deduce some corollaries.

1. In this section we give some properties of representable functors and smooth morphisms.

Let $R$ be a commutative ring; if $F:($ comm. $R$-alg.) $\rightarrow$ (sets) is a functor we will say shortly that $F$ is a sheaf if $F$ is a sheaf of sets on the category of affine schemes over Spec $R$ in the Zariski topology ([1] Def. 0.1 and 0.2).

Proposition 1. Let $F:($ comm. $R$-alg.) $\rightarrow$ (sets) be a functor and suppose that $F$ is a sheaf. Suppose that there exists a family $\left[f_{i}\right]_{i \in I}$ of elements of $R$ generating the unity ideal in $R$, such that the functor $F_{i}$ : (comm. $R_{f_{i}}$-alg.) $\rightarrow$ (sets) induced by $F$ is representable for all $i \in I$; then $F$ is representable. 
Proof. The proof is straightforward and we omit it.

Now we recall the definition of smooth $R$-algebra.

Definition 1. Let $U$ be a commutative $R$-algebra. We say that $U$ is smooth if

(a) $U$ is of finite presentation.

(b) $U$ is formally smooth, i.e. for every commutative $R$-algebra $S$, for every nilpotent ideal $I$ of $S$, and for every $R$-homomorphism $U \rightarrow S / I$, there exists an $R$-homomorphism $U \rightarrow S$ such that the diagram

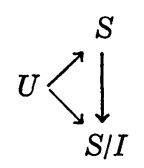

commutes.

Proposition 2. Let $U$ be a commutative $R$-algebra of finite presentation and $S$ a faithfully flat commutative $R$-algebra; then $U$ is a smooth $R$-algebra if and onlf if $U \otimes S$ is a smooth $S$-algebra.

Proof. See [5] Corollary 17.7.2.

Proposition 3. Let $U$ be a commutative $R$-algebra of finite presentation; if for every prime ideal $\mathfrak{p}$ of $R, U_{p}$ is a smooth $R_{p}$-algebra, then $U$ is a smooth $R$-algebra.

Proof. Let $\mathfrak{P}$ be a prime ideal of $U$ and let $\mathfrak{p}=\mathfrak{\beta} \cap R$. $U_{\mathfrak{p}}$ is a smooth $R_{p}$-algebra by hypothesis and it is easy to prove that $U_{\beta}$ is a formally smooth $U_{p}$-algebra. Hence $U_{p}$ is a formally smooth $R_{\mathrm{p}}$-algebra and, by [5] Th. 17.5.1, $U$ is a smooth $R$-algebra.

2. In this section we consider the functor $F_{1}$ defined as follows. Let $A$ and $A^{\prime}$ be two Azumaya $R$-algebras; let a be an ideal of $R$ and suppose that

$$
A / \mathfrak{a} A \approx A^{\prime} / \mathfrak{a} A^{\prime}
$$

For every commutative $R$-algebra $S$, define

$$
F_{1}(S)=\operatorname{Isom}_{S-\text { alg }}\left(A \otimes S, A^{\prime} \otimes S\right)
$$

i.e. $F_{1}(S)$ is the set of isomorphisms of the $S$-algebra $A \otimes S$ onto $A^{\prime} \otimes S . \quad$ It is easy to see that $F_{1}$ is a sheaf. The functor $F_{1}$ satisfies the following properties.

(1) $F_{1}$ is representable. 
By Proposition 1 we can suppose that $A$ and $A^{\prime}$ are free as $R$-modules and with the same rank $n$, because of the hypothesis $A / \mathfrak{a} A \approx A^{\prime} / \mathfrak{a} A^{\prime}$. Let $\left\{e_{i}\right\}$ and $\left\{e_{i}^{\prime}\right\}, i=1, \cdots, n$, be bases for $A$ and $A^{\prime}$ respectively and let

$$
e_{i} e_{j}=\sum_{k} m_{i j k} e_{k}, \quad e_{i}^{\prime} e_{j}^{\prime}=\sum_{k} m_{i j k}^{\prime} e_{k}^{\prime}
$$

be the multiplication laws in $A$ and $A^{\prime}$ respectively. Let $\varphi: A \otimes S \rightarrow$ $A^{\prime} \otimes S$ be an isomorphism; we can write

$$
\varphi\left(e_{i}\right)=\sum_{j} x_{i j} e_{j}^{\prime}, \quad x_{i j} \in S
$$

where the $x_{i j}$ 's must satisfy the following conditions:

(a) since $\varphi$ must satisfy $\varphi\left(e_{i} e_{j}\right)=\varphi\left(e_{i}\right) \varphi\left(e_{j}\right)$ we have

$$
\sum_{k} m_{i j k} x_{k t}=\sum_{k l} m_{k l t}^{\prime} x_{i t} x_{j l}
$$

for all $i, j, t=1, \cdots, n$.

(b) $\operatorname{det}\left(x_{i j}\right)$ is invertible in $S$.

Then consider the ring $R\left[\cdots, X_{i j}, \cdots\right]$ where the $X_{i j}$ 's $(i, j=$ $1, \cdots, n)$ are indeterminate and let

$$
f_{i j t}=\sum_{k} m_{i j k} X_{k t}-\sum_{k l} m_{k l t}^{\prime} X_{i k} X_{j l}
$$

and

$$
d=\operatorname{det}\left(X_{i j}\right)
$$

We set

$$
U_{1}=\left(\frac{R\left[\cdots, X_{i j}, \cdots\right]}{\left(\cdots, f_{i j t}, \cdots\right)}\right)_{d}
$$

and define the isomorphism

$$
\varphi: A \otimes U_{1} \longrightarrow A^{\prime} \otimes U_{1}
$$

by

$$
\varphi\left(e_{i}\right)=\sum_{j} X_{i j} e_{j}^{\prime}
$$

It is immediate to see that the couple $\left(U_{1}, \varphi\right)$ represents the functor $F_{1}$.

(2) The $R$-algebra $U_{1}$ which represents $F_{1}$ is smooth.

(a) By the definition of $U_{1}$ we have that $U_{1}$ is locally of finite presentation, hence $U_{1}$ is of finite presentation ([4] Prop. 1.4.6).

(b) To prove that $U_{1}$ is formally smooth, by Prop. 3 we can 
suppose $R$ local ring. Consider the strict henselization $\widetilde{R}$ of $R$; it is known that, if $\mathfrak{m}$ is the maximal ideal of $R$, then $\mathfrak{m} \widetilde{R}$ is the maximal ideal of $\widetilde{R}$ and the residue field $\Omega$ of $\widetilde{R}$ is a separable closure of the residue field $k$ of $R$ ([11], Chap. VIII $\S 2$ ). We have $A \otimes \Omega \simeq M_{r}(\Omega)$, i.e. the full matrix algebra of rank $r$ over $\Omega$ ([9], Chap. III, Cor. $6.3)$; by this we have

$$
A \otimes \widetilde{R} \simeq M_{r}(\widetilde{R})
$$

([3] Cor. 5.6).

By Proposition 2 we can suppose that

$$
A \simeq M_{r}(R) \simeq A^{\prime}
$$

then $U_{1}$ represents the functor

$$
\text { Aut }\left(M_{r}\right):(\text { comm. } R \text {-alg. }) \longrightarrow(\text { sets })
$$

defined by

$$
\underline{\operatorname{Aut}}\left(M_{r}\right)(S)=\operatorname{Aut}_{S \text {-alg }}\left(M_{r}(S)\right) \text {. }
$$

We must prove that, if $I$ is a nilpotent ideal of $S$, the map

$$
\operatorname{Aut}_{S \text {-alg }}\left(\left(M_{r}(S)\right) \longrightarrow \operatorname{Aut}_{S / I \text {-alg }}\left(M_{r}(S / I)\right)\right.
$$

is surjective.

This is an immediate consequence of the following proposition, because there is a bijection between

$$
\operatorname{Aut}_{S-\mathrm{alg}}\left(M_{r}(S)\right)
$$

and the set of all systems $\left\{e_{i j}\right\}(i, j=1, \cdots, r)$ of matrix units in $M_{r}(S)$.

Proposition 4. Let $(S, I)$ be an henselian couple and $C$ a finite S-algebra. If $\left\{\bar{e}_{i j}\right\}(i, j=1, \cdots, r)$ is a system of matrix units in $C / I C$, then $\left\{\bar{e}_{i j}\right\}$ can be lifted to a system $\left\{e_{i j}\right\}$ of matrix units in $C$.

Proof. The proof is the same as in [3] Th. 3.3.

3. In this section we consider the functor $F_{2}$ defined as follows. Let $P$ be a finite projective $R$-module and, for every commutative $R$-algebra $S$, define $F_{2}(S)=$ set of multiplication laws $m$ which can be defined on $S \otimes P$ such that $(S \otimes P, m)$ is an Azumaya $S$-algebra. Note that $F_{2}$ is a sheaf: this is an easy consequence of the fact that the property of being an Azumaya $R$-algebra is a local property on Spec $R\left([9]\right.$, Chap. III, Th. 6.6). The functor $F_{2}$ satisfies the following properties. 
(1) $F_{2}$ is representable.

By Proposition 1 we can suppose that $P$ is a free $R$-module of rank $n$. Let $\left\{e_{i}\right\}(i=1, \cdots, n)$ be a basis for $P$. A multiplication law on $P \otimes S$ is defined by

$$
e_{i} e_{j}=\sum_{k} m_{i j k} e_{k}, \quad m_{i j k} \in S
$$

where the elements $m_{i j k}$ must satisfy the following properties. By the associative law $\left(e_{i} e_{j}\right) e_{k}=e_{i}\left(e_{j} e_{k}\right)$ we have

$$
\sum_{l}\left(m_{i j l} m_{l k t}-m_{j k l} m_{i l t}\right)
$$

for all $i, j, k, t=1, \cdots, n$.

Let $1=\sum_{i} x_{i} e_{i}$ be the identity element; we have

$$
\sum_{i} x_{i} m_{i j k}=\sum_{i} x_{i} m_{j i k}=\delta_{i k}
$$

for all $i, k=1, \cdots, n$.

In order to express the condition that $(P \otimes S, m)$ is an Azumaya $S$-algebra, we recall the following proposition.

Proposition 5. Let $A$ be an R-algebra and suppose that, as $R$-module, $A$ is free of rank $n$; let $\left\{e_{i}\right\}(i=1, \cdots, n)$ be a basis. Then $A$ is an Azumaya R-algebra if and only if the matrix $\left(a_{i j}\right)$, defined by $a_{i j}=e_{j} e_{i}$, is an invertible matrix in the ring $M_{n}(A)$.

Proof. See [2] Theorem 12.

Then if we denote by $\left(b_{k l}\right)=\left(\sum_{t} m_{k l t}^{\prime} e_{t}\right)$ the inverse matrix of $\left(a_{i j}\right)=\left(\sum_{s} m_{j i s} e_{s}\right)$, we have

$$
\sum_{j k t} m_{j i k} m_{k t s} m_{j l t}^{\prime}=\delta_{i l} x_{s}
$$

for all $i, l, s=1, \cdots, n$.

Then consider the ring

$$
R\left[\cdots, X_{i}, \cdots ; \cdots, Y_{i j k}, \cdots ; \cdots, Y_{i j k}^{\prime}, \cdots\right]
$$

where the $X_{i}^{\prime}$ 's, $Y_{i j k}$ 's, $Y_{i j k}^{\prime}$ 's are indeterminate $(i, j, k=1, \cdots, n)$. Set $f_{i j k t}=\sum_{l}\left(Y_{i j l} Y_{l k t}-Y_{j k l} Y_{i l t}\right)$

$$
\begin{gathered}
g_{j k}=\sum_{i} X_{i} Y_{i j k}-\delta_{j k}, \quad g_{j k}^{\prime}=\sum_{i} X_{i} Y_{j i l t}-\delta_{j k} \\
h_{i l s}=\sum_{j k t} Y_{j i k} Y_{k t s} Y_{j l t}^{\prime}-\delta_{i l} X_{s}
\end{gathered}
$$

and set

$$
U_{2}=\frac{R\left[\cdots, X_{i}, \cdots ; \cdots, Y_{i j k}, \cdots ; \cdots, Y_{i j k}^{\prime}, \cdots\right]}{\left(\cdots, f_{i j k t}, \cdots ; \cdots, g_{j k}, \cdots ; \cdots, g_{j k}^{\prime}, \cdots, h_{i l s}, \cdots\right)} .
$$


Define on $P \otimes U_{2}$ a multiplication law $m$ by

$$
e_{i} e_{j}=\sum_{k} X_{i j k} e_{k} ;
$$

then it is easy to see that $\left(U_{2}, m\right)$ represents $F_{2}$

(2) The R-algebra $U_{2}$ which represents $F_{2}$ is smooth.

(a) As with the algebra $U_{1}, U_{2}$ is of finite presentation.

(b) To see that $U_{2}$ is formally smooth, consider the following proposition.

Proposition 6. Let $S$ be a commutative $R$-algebra and $I$ a nilpotent ideal of $S$; then if $\bar{A}$ is an Azumaya $S /$ I-algebra, there exists an Azumaya S-algebra $A$ such that $A / I A \simeq \bar{A}$.

First we prove that the proposition implies $U_{2}$ formally smooth, i.e. the map $F_{2}(S) \rightarrow F_{2}(S / I)$ surjective. Let $\bar{m} \in F_{2}(S / I)$; call $\bar{A}$ the algebra $(P \otimes S / I, \bar{m})$. By Prop. 6 there exists an Azumaya $S$-algebra $A$ such that $A / I A \simeq \bar{A}$. Call $Q$ the $S$-module underlying to $A ; Q$ is finite and projective and $Q / I Q \simeq P \otimes S / I$. Since $Q$ is projective the above isomorphism lifts to an $S$-module homomorphism $\varphi: Q \rightarrow P \otimes S$ and it is easy to prove that $\varphi$ is an isomorphism. Hence the multiplicative structure on $A$ is carried by $\varphi$ to a multiplication $m$ on $P \otimes S$ whose image in $F_{2}(S / I)$ is $\bar{m}$.

Proof of Proposition 6. We can suppose that $\bar{A}$, as a projective $S / I$-module has constant rank $n$ (by [9] Chap. I. Lemma 6.3 and [3] Cor. 3.2). It is known that there exists a faithfully flat étale extension $\overline{S^{\prime}}$ of $\bar{S}=S / I$ such that

$$
\bar{A} \otimes \bar{S}^{\prime} \simeq M_{r}\left(\bar{S}^{\prime}\right)
$$

with $r^{2}=n$ ([9] Chap. III Cor. 6.3).

By a known theorem ([11] Chap. V, Th. 4) there exists an étale $S$-algebra $S^{\prime}$ such that $S^{\prime} / I S^{\prime} \simeq \bar{S}^{\prime}$ and it is easy to see that $S^{\prime}$ is faithfully flat $S$-algebra. Now recall that, if $S^{\prime}$ is a faithfully flat extension of $S$, the isomorphism classes of Azumaya $S$-algebras $A$ such that

$$
A \otimes S^{\prime} \simeq M_{r}\left(S^{\prime}\right)
$$

are classified by

$$
H^{1}\left(S^{\prime} / S, \text { Aut }\left(M_{r}\right)\right)
$$

where Aut $\left(M_{r}\right):$ (comm. $S$-alg.) $\rightarrow$ (groups) is the functor defined before ([9] Chap. II, Rem. 8.2). Then the Proposition 6 follows from the lemma. 
Lemma. Let $S^{\prime}$ be a faithfully flat extension of $S, I$ a nilpotent ideal of $S, F:$ (comm. $S$-alg.) $\rightarrow$ (groups) a functor represented by a smooth S-algebra. Let $\bar{S}=S / I, \bar{S}^{\prime}=S^{\prime} / I S^{\prime}$ and $\bar{F}:$ (comm. $S / I$-alg.) $\rightarrow$ (groups) be the functor induced by $F$. Then the canonical map

$$
H^{1}\left(S^{\prime} / S, F\right) \longrightarrow H^{1}\left(\bar{S}^{\prime} / \bar{S}, \bar{F}\right)
$$

is bijective.

Proof. [7] Lemma 8.1.8, page 404.

4. In this section we prove the theorem enunciated in the introduction and deduce some corollaries.

First we recall a result on henselian couples.

THeOREM 1. Let $(R, \mathfrak{a})$ be an henselian couple and $U$ a smooth $R$-algebra; then the canonical map

$$
\operatorname{Hom}_{R \text {-alg }}(U, R) \longrightarrow \operatorname{Hom}_{R \text {-alg }}(U, R / \mathfrak{a})
$$

is surjective.

Proof. See [8] Theorem 1.8.

Now we are able to prove the following propositions.

Proposition 7. Let $(R, \mathfrak{a})$ be an henselian couple and $A, A^{\prime}$ two Azumaya $R$-algebras such that $A / \mathfrak{a} A \simeq A^{\prime} / \mathfrak{a} A$; then $A \simeq A^{\prime}$.

Proof. By Theorem 1 and $\S 2$.

Proposition 8. Let $(R, \mathfrak{a})$ be an henselian couple and $\bar{A}$ an Azumaya R/a-algebra; then there exists an Azumaya $R$-algebra $A$ such that $A / \mathfrak{a} A \simeq \bar{A}$.

Proof. Let $\bar{P}$ be the finite projective $R / \mathfrak{a}$-module underlying to $\bar{A}$; then by [3] Theorem 4.1 there exists a finite projective $R$-module $P$ such that $P / \mathfrak{a} P \simeq \bar{P}$. Then the proposition follows from Theorem 1 and $\S 3$.

TheOREm 2. Let $(R, \mathfrak{a})$ be an henselian couple and let $\mathscr{P}(R)$ be the set of isomorphism classes of Azumaya $R$-algebras. Then the canonical map

$$
\mathscr{P}(R) \longrightarrow \mathscr{P}(R / \mathfrak{a})
$$

is bijective. 
Proof. By Propositions 7 and 8.

Corollary 1. Let $(R, a)$ be an henselian couple; then the canonical homomorphism

$$
\mathscr{B} z(R) \longrightarrow \mathscr{B}_{2}(R / \mathfrak{a})
$$

between the Brauer groups is an isomorphism.

Proof. The injectivity is in [3] Proposition 5.7; the surjectivity follows from Theorem 2.

Corollary 2. Let $(R, a)$ be an henselian couple and let

$$
G: \text { (Azumaya } R \text {-alg.) } \longrightarrow \text { (Azumaya } R / \mathfrak{a} \text {-alg.) }
$$

be the functor defined by $G(A)=A / a A$ for every Azumaya $R$-algebra $A$. Then $G$ is essentially bijective and full, but, if $\mathfrak{a} \neq(0)$, is not faithful.

Proof. $G$ is essentially bijective means exactly what we proved in Theorem 2. In order to prove that $G$ is full consider two Azumaya $R$-algebras $A$ and $A^{\prime}$ and define the functor

$$
F^{\prime}:(\text { comm. } R \text {-alg.) } \longrightarrow \text { (sets) }
$$

by

$$
F^{\prime}(S)=\operatorname{Hom}_{S \text {-alg }}\left(A \otimes S, A^{\prime} \otimes S\right) .
$$

As with the functor $F_{1}$ we can prove that $F^{\prime}$ is represented by an $R$-algebra $U^{\prime}$ of finite presentation.

To prove that $U^{\prime}$ is a smooth $\mathrm{R}$-algebra we can suppose, as with the algebra $U_{1}, A \simeq M_{n}(R)$ and $A^{\prime} \simeq M_{m}(R)$. Now observe that, if $\varphi \in F^{\prime}(S)$ and $\left\{e_{i j}\right\}(i, j=1, \cdots, n)$ is a system of matrix units in $A$, then $\left\{\varphi\left(e_{i j}\right)\right\}$ is a system of matrix units in $A^{\prime}$, hence we have

$$
\begin{aligned}
& F^{\prime}(S)=\varnothing \text { if } m \neq n . \\
& F^{\prime}(S)=\operatorname{Aut}_{S \text {-alg }}\left(M_{n}(S)\right) \text { if } m=n .
\end{aligned}
$$

Hence $U^{\prime}$ is a smooth $R$-algebra and by Theorem 1 we have that $G$ is full.

Now let $a \in \mathfrak{a}, a \neq 0$. Consider the inner automorphism $\alpha$ of $M_{2}(R)$ given by the element

$$
\left(\begin{array}{cc}
1+a & 0 \\
0 & 1
\end{array}\right) \in M_{2}(R)
$$

the induced automorphism $\bar{\alpha}$ of $M_{2}(R / \mathrm{a})$ is the identity automorphism 
while $\alpha$ is not the identity automorphism of $M_{2}(R)$. This proves that $G$ is not faithful.

Now suppose $R$ connected and recall that two Azumaya $R$-algebras $A$ and $A^{\prime}$ are said to be stable isomorphic if there exist integers $m$ and $n$ such that

$$
M_{n}(A) \simeq M_{m}\left(A^{\prime}\right) \text {. }
$$

Denote by $\mathscr{K} \mathscr{P}(R)$ the set of stable isomorphism classes of Azumaya $R$-algebras ([6] Remark 1.8).

CoROllaRY 3. Let $(R, a)$ be an henselian couple and suppose that $R / a$ is connected. Then the canonical map

$$
\mathscr{K} \mathscr{P}(R) \longrightarrow \mathscr{K} \mathscr{P}(R / \mathfrak{a})
$$

is bijective.

Proof. First we observe that if $R / \mathfrak{a}$ is connected then $R$ is connected. Now we show that $M_{n}(A)$ is an Azumaya $R$-algebra, if $A$ is an Azumaya $R$-algebra: in fact we know that there exists a faithfully flat extension $S$ of $R$ such that $A \otimes S \simeq M_{r}(S)$; then $M_{n}(A) \otimes S \simeq M_{n \times r}(S)$, i.e. $M_{n}(A)$ is an Azumaya $R$-algebra. Then the Corollary 3 follows from the Propositions 7 and 8.

\section{REFERENCES}

1. M. Artin, Grothendieck Topology, Harvard University 1962.

2. G. Azumaya, On maximally central algebras, Nagoya Math. J., 2 (1951), 119-150.

3. S. Greco, Algebras, over non local Hensel rings, II, J. of Algebra, 13 (1969), 48-56.

4. A. Grothendieck-J. Dieudonné, Eléments de géométric algébrique, Cap. IV partie 1 n. 20, 1964 .

5. — Eléments de géométrie algébrique, Cap. IV partie 4 n. 32, 1967.

6. A. Grothendieck, Le group de Brauer I, Dix exposés sur la cohomologie des schémas, North Holland, Amsterdam, 1968.

7. A. Grothendieck-M. Demazure, Schémas in Groupes III, Lecture Notes in Math. n. 153, Spring-Verlag, Berlin, 1970.

8. L. Gruson, Une propriété des couples henséliennes, Colloque d'Algèbra Comm. Exp. 10, Rennes 1972, Publ. des Sem. Math. de l'Univ. de Rennes.

9. M. A. Knus-M. Ojanguren, Theorie de la Descente et Algèbres d'Azumaya, Lecture Notes in Math. n. 389, Springer-Verlag, Berlin, 1974.

10. J. P. Lafon, Anneaux henséliens, Bull. Soc. Math. France, 91 (1963), 77-107.

11. M. Reynaud, Anneaux locaux henséliens, Lecture Notes in Math. n. 169, SpringerVerlag, Berlin, 1970.

Received April 16, 1975, and in revised form June 20, 1975. This research was done during the academic year 1974-75, when the author was a visitor at Brandeis University, with financial support of the Italian CNR.

BRANDEIS UNIVERSITY

AND

Seminario Matematico, Università di Catania, Corso Italia 55, 95129 Catania, Italy 



\section{PACIFIC JOURNAL OF MATHEMATICS}

\section{EDITORS}

RICHARD ARENS (Managing Editor) University of California

Los Angeles, California 90024

\section{R. A. BEAUMONT}

University of Washington Seattle, Washington 98105
J. DugundjI Department of Mathematics University of Southern Californı Los Angeles, California 90007

D. Gilbarg AND J. Milgram Stanford University Stanford, California 94305

\section{ASSOCIATE EDITORS}

E. F. BECKENBACH

B. H. NeUMANN

F. WOLF

K. YosHidA

\section{SUPPORTING INSTITUTIONS}

UNIVERSITY OF BRITISH COLUMBIA CALIFORNIA INSTITUTE OF TECHNOLOGY UNIVERSITY OF CALIFORNIA MONTANA STATE UNIVERSITY UNIVERSITY OF NEVADA NEW MEXICO STATE UNIVERSITY OREGON STATE UNIVERSITY UNIVERSITY OF OREGON OSAKA UNIVERSITY

\author{
UNIVERSITY OF SOUTHERN CALIFORNIA \\ STANFORD UNIVERSITY \\ UNIVERSITY OF TOKYO \\ UNIVERSITY OF UTAH \\ WASHINGTON STATE UNIVERSITY \\ UNIVERSITY OF WASHINGTON \\ AMERICAN MATHEMATICAL SOCIETY \\ NAVAL WEAPONS CENTER
}




\section{Pacific Journal of Mathematics}

\section{Vol. 61, No. $1 \quad$ November, 1975}

Jiří Adámek, V. Koubek and Věra Trnková, Sums of Boolean spaces represent every

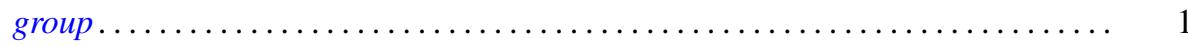

Richard Neal Ball, Full convex l-subgroups and the existence of $a^{*}$-closures of

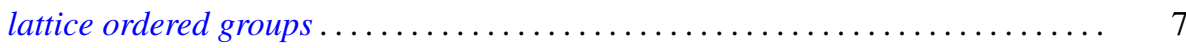

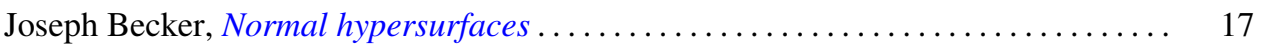

Gerald A. Beer, Starshaped sets and the Hausdorff metric . . . . . . . . . . . . . 21

Dennis Dale Berkey and Alan Cecil Lazer, Linear differential systems with

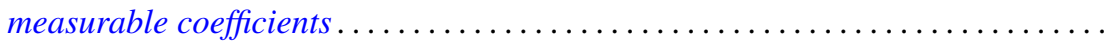

Harald Boehme, Glättungen von Abbildungen 3-dimensionaler

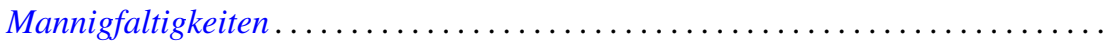

Stephen LaVern Campbell, Linear operators for which $T^{*} T$ and $T+T^{*}$

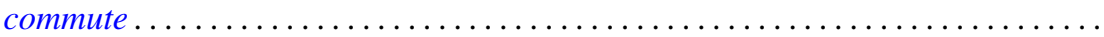

H. P. Dikshit and Arun Kumar, Absolute summability of Fourier series with

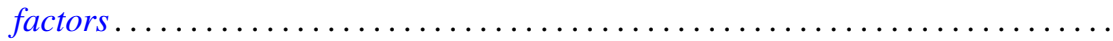

Andrew George Earnest and John Sollion Hsia, Spinor norms of local integral

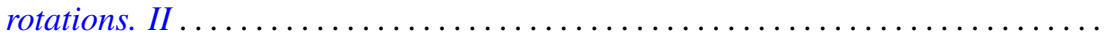

Erik Maurice Ellentuck, Semigroups, Horn sentences and isolic structures .........

Ingrid Fotino, Generalized convolution ring of arithmetic functions . . . . . . . . . . .

Michael Randy Gabel, Lower bounds on the stable range of polynomial rings .......

Fergus John Gaines, Kato-Taussky-Wielandt commutator relations and

characteristic curves

Theodore William Gamelin, The polynomial hulls of certain subsets of $C^{2}$

R. J. Gazik and Darrell Conley Kent, Coarse uniform convergence spaces. . .

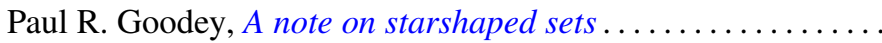

Eloise A. Hamann, On power-invariance

M. Jayachandran and M. Rajagopalan, Scattered compactification for $N \cup\{P\}$. . .

V. Karunakaran, Certain classes of regular univalent functions .

John Cronan Kieffer, A ratio limit theorem for a strongly subadditive set function in a locally compact amenable group .................

Siu Kwong Lo and Harald G. Niederreiter, Banach-Buck measure, density, and uniform distribution in rings of algebraic integers ........

Harold W. Martin, Contractibility of topological spaces onto metric spaces ....

Harold W. Martin, Local connectedness in developable spaces .

A. Meir and John W. Moon, Relations between packing and covering numbers of a tree.

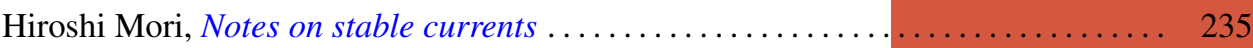

Donald J. Newman and I. J. Schoenberg, Splines and the logarithmic function . . . . 241

M. Ann Piech, Locality of the number of particles operator....

Fred Richman, The constructive theory of $K T$-modules .......

Gerard Sierksma, Carathéodory and Helly-numbers of

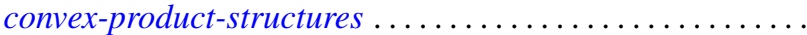

Raymond Earl Smithson, Subcontinuity for multifunctions .... . . 